\title{
Optimal Investment Policy for an Insurer with Ambiguity Aversion: Maximizing Exponential Utility of Terminal Wealth
}

\author{
Bing Liu \\ School of Insurance. \\ Central University of Finance and Economics
}

Cuixia Chen

Hebei Finance University

\begin{abstract}
In this paper, we consider the optimal investment problem for an insurer who worries about the possibility of model misspecification. Without loss of generality, we assume there are only one risky asset and one risk-free asset in the financial market. With consideration of the existence of ambiguity, we aim to obtain the optimal investment policy with maximizing the expected utility of terminal wealth. By the dynamic optimal principle, we obtain closed-form solutions of the optimal investment policy and value function. Furthermore, we find that our study contains some results which are investigated in Browne (1995). We can see that if the insurer is ambiguity aversion, he will appear more conservative about the risky asset.
\end{abstract}

Keywords: Ambiguity aversion; optimal investment; expected utility; penalty function; relative entropy

\section{INTRODUCTION}

As an important way of benefit by an insurance company, the investment problem has been extensively studied in mathematical insurance literature. For example, Browne (1995) analysed the optimal investment policies with maximizing exponential utility of terminal wealth and minimizing the probability of ruin; Hipp and Plum $(2000,2003)$ considered the optimal investment problems for insurers and individuals respectively. In addition, many researchers not only investigate the optimal investment problem but also combine other optimal control problems. Schmidli (2001, 2002), Irgens and Paulson (2004), Bai and Guo (2008), Zhang et al. (2009) and Liang et al. (2011) considered the optimal investment problem combining with reinsurance. Some researchers also investigated the investment problem with considering the optimal dividends problem, for example, see Paulsen and Gjessing (1997), Azcue and Muler (2010). Intuitively, the optimal investment problem paly a crucial role in actuarial science. In this paper, we study the optimal investment problem for an insurance company.

In previous studies, the researchers often aim to obtain the optimal policies under a probability measure $P$. A basic assumption behind these problems is that the insurer knows exactly about the true probability measure $P$. However, some researchers have argued that the assumption is too strong. We know that the probability measure $P$ is constructed by the insurer's limited information. In other words, the insurance company estimates $P$ through a mass of data and various technologies. Obviously, $P$ may be misspecification error. Hence, the insurer should be allowed to consider the optimal policies with considering alternative probability measures. In this paper, we consider the optimal investment and premium control problem for insurers in a diffusion model who worries about the model misspecification. Actually, some scholars have paid attention to the model misspecification problems, for 
example, see Uppal and Wang (2003), Maenhout (2004), Zhang and Siu (2009), Yi et al. (2013), Zeng et al. (2016). In our work, we use a diffusion model to describe the surplus of an insurance company. Based on this diffusion model and considering the existence of model ambiguity, we obtain the closed-form solutions of optimal investment policy and value function. We compare the results with Browne's (1995).

The remainder of this paper is as follows. In Section 2, we give the model used in this paper, describe the objective function in Browne (1995). In Section 3, we discuss the existence of ambiguity and present the objective function. In Section 4, we obtain the closed-form solutions of optimal policy and value function for the problem.

\section{THE MODEL}

We describe the surplus of an insurance company at time $t$ by $S(t)$

$$
\mathrm{d} S(t)=\alpha \mathrm{d} t+\beta \mathrm{d} B_{1}(t), \quad S(0)=x
$$

where $>0,>0 ; B_{1}(t)$ is a standard Brownian motion. We consider there is only one riskfree asset (a bond) and one risky asset (a stock) in a financial market. We assume the price process $P_{0}(t)$ of the risk-free asset satisfies

$$
\mathrm{d} P_{0}(t)=r P_{0}(t) \mathrm{d} t
$$

where $r \quad 0$ is the risk-free interest rate. Furthermore, the price process $P_{1}(t)$ of the risky asset satisfies the standard geometric Brownian motion:

$$
\mathrm{d} P_{1}(\mathrm{t})=\mathrm{P}_{1}(t)\left[\mu \mathrm{d} t+\sigma \mathrm{d} B_{2}(t)\right]
$$

where and are positive constants, $B_{2}(t)$ is the standard Brownian motion. To avoid triviality, we assume $>r$. We assume that the dependence of the insurance market and the financial market is described by $0<1$, i.e., $\operatorname{Cov}\left(B_{1}(t), B_{2}(t)\right)=t$, by standard Gaussian linear regression, we have

$$
\mathrm{d} B_{2}(t)=\rho \mathrm{d} B_{1}(t)+\sqrt{1-\rho^{2}} \mathrm{~d} B_{3}(t),
$$

where $B_{3}(t)$ is a standard Brownian motion that is independent of $B_{1}(t)$. Therefore, the (2.3) becomes

$$
\mathrm{d} P_{1}(t)=P_{1}(t)\left[\mu \mathrm{d} t+\sigma \rho \mathrm{d} B_{1}(t)+\sigma \sqrt{1-\rho^{2}} \mathrm{~d} B_{3}(t)\right],
$$

The insurer is allowed to invest in the risky asset or risk-free asset by himself, and the total amount of money invested in the risky asset at time $t$ is denoted as $f_{t}$. Therefore, the wealth process of the insurance company with the investment policy $f$ can be written as

$$
\mathrm{d} S^{f}(t)=\left[r S^{f}(t)+(\mu-r) f_{t}+\alpha\right] \mathrm{d} t+\left(\beta+\sigma \rho f_{t}\right) \mathrm{d} B_{1}(t)+\sigma f_{t} \sqrt{1-\rho^{2}} \mathrm{~d} B_{3}(t),
$$

where $S^{f}(0)=x$. 
Browne (1995) investigated the optimal investment policies by maximizing the expected utility of terminal wealth and minimizing the probability of ruin corresponding a certain probability measure $P$. In Browne (1995), the objective function is

$$
V_{1}(t, x)=\sup _{f} E^{P}\left\{U\left(S^{f}(T)\right) \mid S^{f}(t)=x\right\}
$$

and

$$
V_{2}(x)=\inf _{f} P\left(\tau_{a} \leq \tau_{b} \mid S^{f}(0)=x\right), a<x<b ;
$$

where $E^{P}$ denotes expectation operator under probability measure $P, U$ is a utility function satisfying $U^{\prime}>0, U^{\prime \prime}<0, T<$ is the terminal time, ${ }_{a}=\inf \left\{t: S^{f}(t) \quad a\right\},{ }_{b}=\inf \left\{t: S^{f}(t) \quad b\right\}$, $f$ is an admissible policy. A basic assumption behind this model is that the insurer knows exactly about true probability measure $P$ used in computing above equation. However, some researchers have argued that the assumption is too strong. The insurer should be allowed to consider the optimal policies for model ambiguity. Therefore, there are many investigators have studied the optimal policies with model ambiguity. For example, see Hansen and Sargent (2001), Uppal and Wang (2003). As a result, we will consider the optimal investment policies with model ambiguity. In the following subsection, we present the model ambiguity in our optimal control problems.

\section{MODEL AMBIGUITY}

We know that the probability measure $P$ above is constructed by the insurer's limited information. In other words, the insurance company estimates $P$ through a mass of data and various technologies. We call this $P$ the reference probability or reference model. Obviously, the insurer is sure about the insurance market, while he is not sure about the financial market. Hence, we can see that the $P$ is the right model to insurance market and it may be misspecification error about financial market. Naturally, the insurer would consider alternative models corresponding to financial model, moreover, the alternative won't change the insurance model. In addition, the alternative models considered by insurer should similar to the reference model, so we define the alternative models by a class of probability measures which are equivalent to $P$ and aren't able to change model (2.1):

$$
\mathbb{Q}:=\{Q \mid Q \sim P ; Q \text { isn't able to change model (2.1) }\}
$$

Since $Q \mathbb{U}$ is equivalent to $P$ and isn't able to change model (2.1), applying the Girsanov's theorem ( Klebaner (2008)), $Q$ is sure that satisfies

$$
\frac{\mathrm{d} Q}{\mathrm{~d} P}\left(B_{3}[0, T]\right)=\Lambda(T)
$$

where

$$
\Lambda(t)=\exp \left(\int_{0}^{t} m(s) \mathrm{d} B_{3}(s)-\frac{1}{2} \int_{0}^{t}[m(s)]^{2} \mathrm{~d} s\right)
$$

is a $P$-martingale with filtration $\left\{F_{t}\right\}_{t[0, T]}, m(t)$ is a regular adapted process satisfying Novikov's condition, i.e.,

$$
E^{P}\left[\exp \left(\frac{1}{2} \int_{0}^{T}[m(s)]^{2} \mathrm{~d} s\right)\right]<\infty
$$


By Girsanov's theorem, the standard Brownian motion $B(t)$ under probability measure $P$ can be represented as

$$
\mathrm{d} B_{3}(t)=m(t) \mathrm{d} t+\mathrm{d} B_{3}^{Q}(t)
$$

where $B_{3}^{Q}(t)$ is a standard Brownian motion under probability measure $Q$, furthermore, $B_{1}(t)$ is also a standard Brownian motion under probability measure $Q$. We should derive the wealth process under $Q$. Inserting (3.3) and into (2.5), we have

$$
\begin{aligned}
\mathrm{d} S^{Q}(t)=\left[r S^{Q}(t)\right. & \left.+(\mu-r) f_{t}+\alpha+\sigma f_{t} \sqrt{1-\rho^{2}} m(t)\right] \mathrm{d} t+\left(\beta+\sigma \rho f_{t}\right) \mathrm{d} B_{1}(t) \\
& +\sigma f_{t} \sqrt{1-\rho^{2}} \mathrm{~d} B_{3}^{Q}(t), \quad S^{Q}(0)=x .
\end{aligned}
$$

For the purpose of considering the alternative model $Q$, we need to measure the discrepancy between each alternative model and reference model by using relative entropy. The use of relative entropy is a well-established approach in measuring the discrepancy between $Q$ and $P$. Numerous studies have used relative entropy to measure it, for example, see Uppal and Wang (2003), Maenhout (2004), Yi et al. (2013). The relative entropy between $Q$ and $P$ is given by

$$
\begin{aligned}
H_{[0, t]}(Q \| P)=E_{[0, t]}^{Q}\left[\ln \frac{\mathrm{d} Q}{\mathrm{~d} P}\right] & =E^{Q}\left[\int_{0}^{t} m(s) \mathrm{d} B_{3}(s)-\frac{1}{2} \int_{0}^{t}[m(s)]^{2} \mathrm{~d} s\right] \\
& =E^{Q}\left[\int_{0}^{t} m(s) \mathrm{d} B_{3}^{Q}(s)+\frac{1}{2} \int_{0}^{t}[m(s)]^{2} \mathrm{~d} s\right]
\end{aligned}
$$

Since $B_{3}^{Q}(t)$ is a standard Brownian motion under probability measure $Q$, we have

$$
H_{[0, t]}(Q \| P)=E^{Q}\left[\int_{0}^{t} Z(s) \mathrm{d} s\right]=E^{Q}\left[\int_{0}^{t} \frac{1}{2}[m(s)]^{2} \mathrm{~d} s\right]
$$

where $Z(s)=\frac{1}{2}[m(s)]^{2}$. Hence, $Z(t)$ measures the $H_{[0, t]}(Q \| P)$. If the insurer rejects the reference model $P$ and accepts the alternative model $Q$, a penalty is incurred. Obviously, the larger the $H_{[0, t]}(Q \| P)$ is, the larger the penalty is incurred. It is similar to Uappl and Wang (2003), Maenhout (2004), we formulate robust control problems in the following. The objective of maximizing exponential utility of terminal wealth with ambiguity is set as

$$
V(t, x)=\operatorname{supinf}_{f \in \Pi} E_{t \in \mathbb{Q}}^{Q}\left[\int_{t}^{T} \xi \phi\left(V\left(s, S^{Q}(s)\right)\right) Z(s) \mathrm{d} s+U\left(S^{Q}(T)\right)\right],
$$

where $E_{t, x}^{Q}[]=E^{Q} \quad \mid S^{Q}(t)=x, \quad()>0$ is a standardization function that converts the penalty to the same order of magnitude as the order of $V(t, x)$, the special form of () is usual selected for analytical simplicity, $>0$ denotes the insurer's confidence on reference model $P$, the larger the is, the insurer has more confidence on $P$, that the is the set of admissible 
policies will be detailed later, the inf term reflects the insurer's aversion to ambiguity, in other words, the insurer is conservative and then he will consider the worst result with ambiguity. In the extreme case, means that the insurer is extremely confident about the reference model and the use of any alternative model will incur the heavily penalty, therefore, the problem (3.6) reduces to (2.6). On the other hand, 0 means that the insurer has no information about the reference model. Hence, we assume $0<<\quad$ in the following study.

\section{MAXIMIZING EXPONENTIAL UTILITY OF TERMINAL WEALTH WITH AMBIGUITY}

Suppose that the insurer has a CARA utility

$$
U(x)=-\frac{1}{\gamma} \exp (-\gamma x)
$$

where $>0$ is a constant absolute risk aversion.

For a Markov control process $f$ and any function $g(t, x) \mathbb{C}^{1,2}$, we define a generator corresponding to (3.4) is denoted as

$$
\begin{aligned}
\nabla^{f} g(t, x)= & \frac{\partial}{\partial t} g(t, x)+\left[r S^{Q}(t)+(\mu-r) f_{t}+\alpha+\sigma f_{t} \sqrt{1-\rho^{2}} m(t)\right] \frac{\partial}{\partial x} g(t, x) \\
& +\frac{1}{2}\left[\beta^{2}+\sigma^{2} f_{t}^{2}+2 \rho \sigma \beta f_{t}\right] \frac{\partial^{2}}{\partial x^{2}} g(t, x) .
\end{aligned}
$$

In addition, we give a definition of the set that contains all admissible policies.

Definition 4.1. We say that $=\left\{f_{t}\right\}_{t[0, T]}$ is an admissible set, if

(i) The process $f=\left\{f_{t}, 0<t<T\right\}$ is a predictable and satisfy that

$$
E^{Q} \int_{0}^{T} f_{s}^{2} \mathrm{~d} s<\infty, T<\infty, Q \in \mathbb{Q} \text {; }
$$

(ii) The stochastic differential equation (3.4) determines a unique strong solution.

We can easily to get that the value function $V(t, x)$ of $(3.6)$ satisfies that $V(t, x)<0$,

$$
\frac{\partial}{\partial x} V(t, x)>0, \frac{\partial^{2}}{\partial x^{2}} V(t, x)<0 \text {. }
$$

According to the technique of dynamic programming principle, $V(t, x)$ satisfies the HamiltonJacobi-Bellman (HJB) equation (see Fleming and Soner (2006))

$$
\operatorname{supinf}_{f \in \Pi}\left\{\nabla^{f} V(t, x)+\frac{1}{2} \xi \phi(V(t, x)) m^{2}\right\}=0
$$

with the boundary condition $V(T, x)=U(x)$.

Theorem 4.1 (Verification Theorem) If $(t, x) \quad \mathbb{C}^{1,2}$ is the solution to HJB equation (4.3) with the boundary condition $(T, x)=U(x)$, then $(t, x)=V(t, x)$.

Proof. The proof is normal. We omit it here. 
Furthermore, as mentioned before, in order to converts the penalty to the same order of magnitude as the order of $V(t, x)$, we choose a suitable form of ( )

$$
\phi(V(t, x))=-V(t, x)
$$

Substituting (4.4) into (4.3), we have

$$
\begin{aligned}
\operatorname{supinf}_{f \in \Pi}\{ & \frac{\partial}{\partial t} V(t, x)+\left[r x+(\mu-r) f_{t}+\alpha+\sigma f_{t} \sqrt{1-\rho^{2}} m\right] \frac{\partial}{\partial x} V(t, x) \\
& \left.+\frac{1}{2}\left[\beta^{2}+\sigma^{2} f_{t}^{2}+2 \rho \sigma \beta f_{t}\right] \frac{\partial^{2}}{\partial x^{2}} V(t, x)-\frac{1}{2} \xi V(t, x) m^{2}\right\}=0
\end{aligned}
$$

Since $\frac{1}{2} V(t, x) m^{2}>0$, in accordance with the first-order conditions, the function $m^{*}$ minimizes the (4.5) that has the following form

$$
m^{*}=\frac{\left(\sigma f_{t} \sqrt{1-\rho^{2}}\right) \frac{\partial}{\partial x} V(t, x)}{\xi V(t, x)} .
$$

Substituting (4.6) into (4.5), yields

$$
\begin{gathered}
\operatorname{supinf}_{f \in \Pi}\left\{\frac{\partial}{\partial t} V(t, x)+\left[r x+(\mu-r) f_{t}+\alpha\right] \frac{\partial}{\partial x} V(t, x)+\frac{\sigma^{2} f_{t}^{2}\left(1-\rho^{2}\right)}{2 \xi V(t, x)}\left[\frac{\partial}{\partial x} V(t, x)\right]^{2}\right. \\
\left.+\frac{1}{2}\left[\beta^{2}+\sigma^{2} f_{t}^{2}+2 \rho \sigma \beta f_{t}\right] \frac{\partial^{2}}{\partial x^{2}} V(t, x)\right\}=0
\end{gathered}
$$

To obtain the optimal policies, we should have the explicit solution to (3.6). With the exponential form of utility function, we speculate that the value function has the following form

$$
V(t, x)=-\frac{1}{\gamma} \exp \{-\gamma[D(t) x+L(t)]\}
$$

where $D(t), L(t)$ is suitable function respect to $t$. Since $V(T, x)=U(x)$, we have $D(T)=1$, $L(T)=0$. Then, we have

$$
\begin{aligned}
& \frac{\partial}{\partial t} V(t, x)=V(t, x)\left\{-\gamma\left[D^{\prime}(t) x+L^{\prime}(t)\right]\right\} \\
& \frac{\partial}{\partial x} V(t, x)=-V(t, x) \gamma D(t) \\
& \frac{\partial^{2}}{\partial x^{2}} V(t, x)=V(t, x) \gamma^{2} D(t)^{2} .
\end{aligned}
$$

Putting (4.9)-(4.11) into (4.7), we have

$$
\sup _{f \in \Pi}\left\{V(t, x)\left[-\gamma\left(D^{\prime}(t) x+L^{\prime}(t)\right)\right]-\left[r x+(\mu-r) f_{t}+\alpha\right] V(t, x) \gamma D(t)\right.
$$




$$
\begin{aligned}
& +\frac{1}{2}\left(\beta^{2}+\sigma^{2} f_{t}^{2}+2 \rho \sigma \beta f_{t}\right) V(t, x) \gamma^{2} D(t)^{2} \\
& \left.+\frac{\sigma^{2} f_{t}^{2}\left(1-\rho^{2}\right)}{2 \xi} V(t, x) \gamma^{2} D(t)^{2}\right\}=0 .
\end{aligned}
$$

Since $V(t, x)<0$, dividing $V(t, x)$ by both sides of (4.12), we have

$$
\begin{aligned}
\inf _{f \in \Pi}\{ & -\gamma\left(D^{\prime}(t) x+L^{\prime}(t)\right)-\left[r x+(\mu-r) f_{t}+\alpha\right] \gamma D(t)+\frac{1}{2}\left(\beta^{2}+\sigma^{2} f_{t}^{2}+2 \rho \sigma \beta f_{t}\right) \gamma^{2} D(t)^{2} \\
+ & \left.\frac{\sigma^{2} f_{t}^{2}\left(1-\rho^{2}\right)}{2 \xi} \gamma^{2} D(t)^{2}\right\}=0
\end{aligned}
$$

According to first-order conditions, we have

$$
f_{t}^{*}=\frac{(\mu-r)-\rho \sigma \beta \gamma D(t)}{\sigma^{2} \gamma D(t)\left(1+\frac{1-\rho^{2}}{\xi}\right)}
$$

Inserting (4.14) into (4.13), we obtain

$$
-\gamma D^{\prime}(t) x-r x \gamma D(t)-\gamma L^{\prime}(t)-\alpha \gamma D(t)+\frac{1}{2} \beta^{2} \gamma^{2} D(t)^{2}-\frac{1}{2} \frac{[(\mu-r)-\rho \sigma \beta \gamma D(t)]^{2}}{\sigma^{2}\left(1+\frac{1-\rho^{2}}{\xi}\right)}=0 .
$$

Equation (4.15) holds, if the following equations are satisfied:

$$
\begin{aligned}
& -\gamma D^{\prime}(t) x-r x \gamma D(t)=0 \\
& -\gamma L^{\prime}(t)-\alpha \gamma D(t)+\frac{1}{2} \beta^{2} \gamma^{2} D(t)^{2}-\frac{1}{2} \frac{[(\mu-r)-\rho \sigma \beta \gamma D(t)]^{2}}{\sigma^{2}\left(1+\frac{1-\rho^{2}}{\xi}\right)}=0 .
\end{aligned}
$$

By the boundary condition $D(T)=1$ and $L(T)=0$, we have

$$
\begin{aligned}
& D(t)=\exp (r(T-t)), \\
& L(t)=\left\{\begin{array}{cc}
-\left(\alpha-\frac{(\mu-r) \rho \beta}{\sigma\left(1+\frac{1-\rho^{2}}{\xi}\right)}\right)\left(1-\frac{1}{r} e^{r(T-t)}\right)+\frac{1}{2} \beta^{2} \gamma\left(1-\frac{\rho^{2}}{\left.1+\frac{1-\rho^{2}}{\xi}\right)}\right)\left(\frac{1}{2 r}-e^{2 r(T-t)}\right)+\frac{1}{2} \frac{(\mu-r)^{2}}{\sigma^{2} \gamma\left(1+\frac{1-\rho^{2}}{\xi}\right)}(T-t), & r>0 ; \\
{\left[\alpha-\frac{1}{2} \beta^{2} \gamma+\frac{1}{2} \frac{(\mu-\rho \sigma \beta \gamma)^{2}}{\sigma^{2} \gamma\left(1+\frac{1-\rho^{2}}{\xi}\right)}\right](T-t),} & r=0 .
\end{array}\right.
\end{aligned}
$$

Substituting (4.16) into (4.14), yields

$$
f_{t}^{*}=\left[\frac{\mu-r}{\sigma^{2} \gamma} e^{-r(T-r)}-\frac{\rho \beta}{\sigma}\right] \frac{1}{1+\frac{1-\rho^{2}}{\xi}} \text {. }
$$


Before we give the follow theorem, we also need to verify that the Novikov's condition is satisfied. In other words, we should guarantee $(T)$ with $m^{*}$ is a P-martingale. Hence, we should have

$$
E\left[\exp \left(\frac{1}{2} \int_{0}^{T}[m(t)]^{2} \mathrm{~d} s\right)\right]<\infty
$$

That is

$$
E\left[\exp \left(\frac{1}{2} \int_{0}^{T}\left[\frac{\sigma f_{t}^{*} \sqrt{1-\rho^{2}} \frac{\partial}{\partial x} V(t, x)}{\xi V(t, x)}\right]^{2} \mathrm{~d} s\right]<<\right.
$$

which equivalent to

$$
E\left[\exp \left(\frac{1}{2} \int_{0}^{T}\left[\frac{\sigma f_{t}^{*} \sqrt{1-\rho^{2}}(-\gamma D(t))}{\xi}\right]^{2} \mathrm{~d} s\right)\right]<\infty,
$$

we know that $f_{t}^{*}<, D(t)<, T<$ and other parameters are constants, therefore (4.18) is satisfied. In the above process, we have proved the following theorem.

Theorem 4.2. For the problem (3.6) with (4.4), if the insurer has a CARA utility function (4.1), we can obtain the optimal policy in the following:

$$
f_{t}^{*}=\left[\frac{\mu-r}{\sigma^{2} \gamma} e^{-r(T-r)}-\frac{\rho \beta}{\sigma}\right] \frac{1}{1+\frac{1-\rho^{2}}{\xi}} .
$$

The corresponding value function can be written as

$$
V(t, x)=-\frac{1}{\gamma} \exp \{-\gamma[D(t) x+L(t)]\}
$$

where $D(t)$ and $L(t)$ are given by (4.16) and (4.17) respectively.

Remark. The optimal investment is verified same as the ones without considering model misspecification when . This is because that the means that the insurer is extremely confident to the reference model $P$. Hence, there is no ambiguity about the reference model and the research problem in this paper reduce to Browne's (1995). Furthermore, the optimal policy is same as the corresponding part in Browne (1995). On the other hand, when $0, f_{t}^{*} \quad 0$. This is because 0 means that the insurer has no information about this finance market. Thus, the insurer will not invest in this finance market. 
Remark. Specially, if the risk-free interest rate $r=0$, the optimal investment policy becomes

$$
f_{t}^{*}=\left[\frac{\mu-r}{\sigma^{2} \gamma}-\frac{\rho \beta}{\sigma}\right] \frac{1}{1+\frac{1-\rho^{2}}{\xi}}
$$

\section{References}

Azcue, P., Muler, N. (2010). Optimal investment policy and dividend payment strategy in an insurance company. Annals of Applied Probability 20(4), 1253-1302.

Bai, L., Guo, J. (2008). Optimal proportional reinsurance and investment with multiple risky assets and noshorting constraint. Insurance Mathematics and Economics 42(3), 968-975.

Browne, S. (1995). Optimal investment policies for a firm with a random risk process: exponential utility and minimizing the probability of ruin. Mathematics of Operations Research 20, 937-958.

Fleming, W.H., Soner, M. (2006). Controlled Markov Processes and Viscosity Solutions, 2nd edition. Springer, New York.

Hansen, L. P., Sargent, T. J. (2001). Robust control and model uncertainty. American Economic Review 91(2), 6066.

Hipp, C., Plum, M. (2000). Optimal investment for insurers. Insurance Mathematics and Economics 27(2), 215-228.

Hipp, C., Plum, M. (2003). Optimal investment for investors with state dependent income, and for insurers. Finance and Stochastics 7(3), 299-321.

Irgens, C., Paulsen, J. (2004). Optimal control of risk exposure, reinsurance and investments for insurance portfolios. Insurance Mathematics and Economics 35(1), 21-51.

Liang, Z., Yuen, K. C., Guo, J. (2011). Optimal proportional reinsurance and investment in a stock market with Ornstein-Uhlenbeck process. Insurance Mathematics and Economics 49(2), 207-215.

Klebaner, F. C. (2008). Introduction to stochastic calculus with applications. IMPERIAL COLLEGE PRESS.

Maenhout, P. J. (2004). Robust portfolio rules and asset pricing. Review of Financial Studies 17(4), 951-983.

Paulsen, J., Gjessing, H.K. (1997). Optimal choice of dividend barriers for a risk process with stochastic return on investments. Insurance: Mathematics and Economics 20, 215-223.

Schmidli, H. (2001). Optimal proportional reinsurance policies in a dynamic setting. Scandinavian Actuarial Journal 1, 55-68.

Schmidli, H. (2002). On minimizing the ruin probability by investment and reinsurance. Annals of Applied Probability 12, 890-907.

Uppal, R., Wang, T. (2003). Model misspecification and underdiversification. The Journal of Finance 58(6), 24652486.

Yi, B., Li, Z., Viens, F. G., Zeng, Y. (2013). Robust optimal control for an insurer with reinsurance and investment under Hestons stochastic volatility model. Insurance Mathematics and Economics 53(3), 601-614.

Zeng, Y., Li, D., Gu, A. (2016). Robust equilibrium reinsurance-investment strategy for a mean-variance insurer in a model with jumps. Insurance Mathematics and Economics 66, 138-152.

Zhang, X., Zhang, K., Yu, X. (2009). Optimal proportional reinsurance and investment with transaction costs, I: Maximizing the terminal wealth. Insurance Mathematics and Economics 44, 473-478. 